PROCEEDINGS OF THE

AMERICAN MATHEMATICAL SOCIETY

Volume 126, Number 2, February 1998, Pages 507-514

S 0002-9939(98)04044-1

\title{
ON UNIQUENESS OF INVARIANT MEANS
}

\author{
M. B. BEKKA \\ (Communicated by Roe Goodman) \\ Dedicated to Professor Eberhard Kaniuth on the occasion of his 60th birthday
}

ABSTRACT. The following results on uniqueness of invariant means are shown:

(i) Let $\mathbb{G}$ be a connected almost simple algebraic group defined over $\mathbb{Q}$. Assume that $\mathbb{G}(\mathbb{R})$, the group of the real points in $\mathbb{G}$, is not compact. Let $p$ be a prime, and let $\mathbb{G}\left(\mathbb{Z}_{p}\right)$ be the compact $p$-adic Lie group of the $\mathbb{Z}_{p}$-points in $\mathbb{G}$. Then the normalized Haar measure on $\mathbb{G}\left(\mathbb{Z}_{p}\right)$ is the unique invariant mean on $L^{\infty}\left(\mathbb{G}\left(\mathbb{Z}_{p}\right)\right)$.

(ii) Let $G$ be a semisimple Lie group with finite centre and without compact factors, and let $\Gamma$ be a lattice in $G$. Then integration against the $G$-invariant probability measure on the homogeneous space $G / \Gamma$ is the unique $\Gamma$-invariant mean on $L^{\infty}(G / \Gamma)$.

\section{INTRODUCTION AND STATEMENT OF THE RESULTS}

Let $G$ be a group acting on a probability measure space $(X, \mu)$ in a measure preserving way. An invariant mean on $L^{\infty}(X)$, the space of the $\mu$-essentially bounded functions on $X$, is a linear functional $m: L^{\infty}(X) \rightarrow \mathbb{C}$ such that $m(1)=1, m(\varphi) \geq 0$ if $\varphi \geq 0$ and $m\left({ }_{g} \varphi\right)=m(\varphi)$ for all $\varphi$ in $L^{\infty}(X)$ and $g$ in $G$, where ${ }_{g} \varphi$ is defined by ${ }_{g} \varphi(x)=\varphi\left(g^{-1} x\right)$. It is clear that integration against $\mu$ defines an invariant mean, also denoted by $\mu$, on $L^{\infty}(X)$.

The question of the uniqueness of $\mu$ as invariant mean is of considerable interest. For instance, the Banach-Ruziewicz problem asks whether the Lebesgue measure $\lambda$ on the $n$-dimensional sphere $S^{n}$ is the unique normalized, finitely additive $S O(n+1)$-invariant measure on all Lebesgue measurable subsets of $S^{n}$. For $n \geq 2$, this is known to be equivalent to the uniqueness of $\lambda$ as $S O(n+1)$-invariant mean on $L^{\infty}\left(S^{n}\right)$ or - what amounts to the same - to the uniqueness of the Haar measure on $S O(n+1)$ as invariant mean on $L^{\infty}(S O(n+1))$. This problem has been solved in the affirmative by Margulis [Ma1] and Sullivan [Sul] in the case $n \geq 4$ and by Drinfeld [Dri] in the remaining cases $n=2, n=3$. From these results one may deduce the following (see [Ma2], Chap. III, 5. Proposition (7)))

Theorem. Let $G$ be a semisimple compact real Lie group. Then the normalized Haar measure on $G$ is the unique $G$-invariant mean on $L^{\infty}(G)$.

Received by the editors May 6, 1996 and, in revised form, August 12, 1996.

1991 Mathematics Subject Classification. Primary 43A07, 22E40.

Key words and phrases. Invariant means on compact groups, $p$-adic groups, Selberg inequality, lattices in semisimple Lie groups, linear algebraic groups.

(C) 1998 American Mathematical Society 
For all of this and much more, we recommend the excellent monographs [Lub], [Sar] and [Wag].

In contrast, nothing seems to be known in the totally disconnected case (compare [Lub], Problem 10.2.1). The first purpose of this note is to prove the analogue of the above theorem for some compact $p$-adic groups like $S L\left(n, \mathbb{Z}_{p}\right)$ or $S p\left(n, \mathbb{Z}_{p}\right)$.

Theorem A. Let $\mathbb{G}$ be an almost simple connected linear algebraic group defined over $\mathbb{Q}$. Assume that $\mathbb{G}(\mathbb{R})$, the group of the real points in $\mathbb{G}$, is not compact. Let $p$ be a prime in $\mathbb{N}$. Then the normalized Haar measure on $\mathbb{G}\left(\mathbb{Z}_{p}\right)$ is the unique invariant mean on $L^{\infty}\left(\mathbb{G}\left(\mathbb{Z}_{p}\right)\right)$, where $\mathbb{Z}_{p}$ is the ring of the $p$-adic integers.

We do not know whether the non-compactness assumption on $\mathbb{G}(\mathbb{R})$ in Theorem $\mathrm{A}$ is necessary. ${ }^{1}$

Recall that a lattice $\Gamma$ in a locally compact group $G$ is a discrete subgroup of $G$ such that the Haar measure on $G$ induces a finite $G$-invariant Borel measure $\mu$ on the homogeneous space $G / \Gamma$. Of course, we may assume that $\mu$ is a probability measure. Our second result shows that $\mu$ is unique as $\Gamma$-invariant mean on $L^{\infty}(G / \Gamma)$ when $\Gamma$ is a lattice in a real semisimple Lie $G$.

Theorem B. Let $G$ be a semisimple Lie group with finite centre and without compact factors. Let $\Gamma$ be a lattice in $G$. Then the $G$-invariant probability measure on $G / \Gamma$ is the unique $\Gamma$-invariant mean on $L^{\infty}(G / \Gamma)$.

The proofs of Theorems A and B depend on an isolation property of the trivial representation of certain discrete groups (see Lemma 1 below). In Theorem A, this property follows from extensions of Selberg's inequality $\lambda_{1} \geq 3 / 16$ for the first eigenvalue of the Laplacian acting on an arithmetic surface $\Gamma(N) \backslash \mathbb{H}$, where $\Gamma(N)$ is a congruence subgroup of $S L(2, \mathbb{Z})$ and $\mathbb{H}$ is the hyperbolic plane (see [EGM], $[\mathrm{LPS}],[\mathrm{BuS}])$. As to the proof of Theorem B, we use some general facts, due to Langlands, on the decomposition of $L^{2}(\Gamma \backslash G)$. Another ingredient is the existence of a spectral gap over 0 of the Laplacian on the locally symmetric space $\Gamma \backslash G / K$ (see $[\mathrm{BoG}],[\mathrm{OsW}])$.

\section{ProOFs}

The following crucial lemma is the link between uniqueness of invariant means and unitary group representation theory. This result, due to V. Losert and H. Rindler [LoR], J. Rosenblatt [Ros], and K. Schmidt [Sch], was used for the solution of the Banach-Ruziewicz problem in [Dri], [Ma1] and [Sul]. For the proof, see e.g. [Lub], Proposition 3.4.1 and the remark after Theorem 3.4.4.

Lemma 1. Let $G$ be a discrete group acting on a probability measure space $(X, \mu)$ and preserving $\mu$. Let $\rho$ denote the natural unitary representation of $G$ on $L^{2}(X)$ and $\rho_{0}$ the restriction of $\rho$ to the invariant subspace

$$
L_{0}^{2}(X)=\left\{f \in L^{2}(X) ; \int_{X} f d \mu=0\right\}
$$

(the orthogonal of the constants on $X$ ). Suppose that $\rho_{0}$ does not weakly contain $1_{G}$, the trivial one-dimensional representation of $G$. Then $\mu$ is the unique invariant mean on $L^{\infty}(X)$.

\footnotetext{
${ }^{1}$ See the note added in proof.
} 
Recall that if $\sigma$ and $\pi$ are unitary representations of the locally compact group $G$, then $\sigma$ is weakly contained in $\pi$ if every positive definite function associated with $\sigma$ is a uniform limit over compacta of sums of positive definite functions associated with $\pi$. In the case where $\sigma=1_{G}$, this amounts to the existence of almost invariant $\pi(G)$-invariant vectors, that is, a net $\left(\xi_{\iota}\right)_{\iota}$ of unit vectors in the Hilbert space of $\pi$ so that $\left\|\pi(g) \xi_{\iota}-\xi_{\iota}\right\| \rightarrow 0$ uniformly on compacta.

Let $\widehat{G}$ denote the unitary dual of $G$, that is, the set of equivalence classes of irreducible unitary representations of $G$. The Fell-Jacobson topology on $\widehat{G}$ is defined by the following closure operation: an irreducible representation $\pi$ is in the closure of a subset $\Pi$ of $\hat{G}$ if and only if $\pi$ is weakly contained in the direct sum $\bigoplus_{\sigma \in \Pi} \sigma$. For all of this, see [Dix], Chap. 18, or [Fe1].

We shall need the following lemma.

Lemma 2. Let $\mathbb{G}$ be a connected semisimple linear algebraic group defined over $\mathbb{Q}$, and let $p$ be a prime in $\mathbb{N}$. Assume that $\mathbb{G}$ is almost simple over $\mathbb{Q}_{p}$ and that $\mathbb{G}(\mathbb{R})$ is not compact. Then the closure of $\mathbb{G}(\mathbb{Z})$ has finite index in $\mathbb{G}\left(\mathbb{Z}_{p}\right)$.

Proof. Let $H$ denote the closure of $\mathbb{G}(\mathbb{Z})$ in $\mathbb{G}\left(\mathbb{Z}_{p}\right)$.

By a result of Borel and Harish-Chandra ([BHC], Theorem 12.3), the subgroup $\mathbb{G}(\mathbb{Z})$ of $H$ is a lattice in $\mathbb{G}(\mathbb{R})$. Since $\mathbb{G}(\mathbb{R})$ is not compact, the Borel-Wang density theorem shows that $H$ is Zariski dense in $\mathbb{G}$ (see [Ma2], Chap. II, (4.4) Corollary). This implies that the Lie algebra of the $p$-adic Lie subgroup $H$ of $\mathbb{G}\left(\mathbb{Q}_{p}\right)$ is $\mathbb{G}$ invariant. Hence, as $\mathbb{G}\left(\mathbb{Q}_{p}\right)$ is almost simple, $H$ is either open or discrete in $\mathbb{G}\left(\mathbb{Q}_{p}\right)$. If $H$ were discrete, then $\mathbb{G}(\mathbb{Z})$ would be a discrete and hence finite subgroup of the compact group $\mathbb{G}\left(\mathbb{Z}_{p}\right)$. This is not the case. Hence, $H$ is open in $\mathbb{G}\left(\mathbb{Z}_{p}\right)$ and, as $\mathbb{G}\left(\mathbb{Z}_{p}\right)$ is compact, it has finite index.

Remark. The assumption that $\mathbb{G}(\mathbb{R})$ is not compact is necessary in the previous lemma. Indeed, $\mathbb{G}(\mathbb{Z})$ is finite when $\mathbb{G}(\mathbb{R})$ is compact.

Proof of Theorem $A$. Set $G=\mathbb{G}\left(\mathbb{Z}_{p}\right)$, and let $\rho_{0}$ denote the restriction to $L_{0}^{2}(G)$ of the left regular representation of $G$ on $L^{2}(G)$. By Lemma 1, it suffices to show that $1_{G}$ is not weakly contained in $\rho_{0}$, when $G$ is viewed as a discrete group.

Let $\Gamma=\mathbb{G}(\mathbb{Z})$. For $N \in \mathbb{N}$, let $\Gamma(N)$ denote the principal congruence subgroup

$$
\Gamma(N)=\{\gamma \in \Gamma ; \gamma \equiv I(\bmod N)\} .
$$

Let $\widehat{\Gamma}_{\text {arith }}$ be the subset of the unitary dual $\widehat{\Gamma}$ consisting of those irreducible unitary representations that factor through some quotient $\Gamma / \Gamma(N)$. The crucial fact is that $1_{\Gamma}$ is isolated in $\widehat{\Gamma}_{\text {arith }}$.

Indeed, $\Gamma=\mathbb{G}(\mathbb{Z})$ is a lattice in $\mathbb{G}(\mathbb{R})([\mathrm{BHC}]$, loc. cit. $)$. Hence, if $\mathbb{G}(\mathbb{R})$ has Kazhdan's property $(\mathrm{T})$, then $\Gamma$ has also property $(\mathrm{T})$ and the claim is clear in this case. In the remaining cases, the claim follows from work by Elstrodt, Grunewald, Mennicke [EGM], Li, Piatestski-Shapiro, Sarnak [LPS], Burger, Sarnak [BuS], when $\mathbb{G}(\mathbb{R})$ is locally isomorphic to $S O(n, 1)$ and from work by $\mathrm{Li}([\mathrm{Li}])$ when $\mathbb{G}(\mathbb{R})$ is locally isomorphic to $S U(n, 1)$. It should be mentioned that this work is a generalization of Selberg's famous inequality $\lambda_{1} \geq 3 / 16$ in the case of $S L(2, \mathbb{R})$ for the lowest eigenvalue $\lambda_{1}$ of the Laplacian on $\Gamma(N) \backslash \mathbb{H}$, where $\mathbb{H}$ is the real hyperbolic plane (see [Sar], (A.2.14)). These results amount to saying that $1_{\mathbb{G}(\mathbb{R})}$ is isolated in 
the spectrum of the direct sum

$$
\bigoplus_{N \in \mathbb{N}} \operatorname{Ind}_{\Gamma(N)}^{\mathbb{G}(\mathbb{R})} 1_{\Gamma(N)}
$$

This implies that $1_{\Gamma}$ is isolated in $\widehat{\Gamma}_{\text {arith }}$ (see [Lub], Theorem 4.3.2; see also [Bro] and $[$ Bur $]$ ).

Now let $\tau$ be the profinite topology on $\Gamma$ defined by the subgroups $\Gamma\left(p^{n}\right), n \in \mathbb{N}$, that is, the group topology on $\Gamma$ for which the $\Gamma\left(p^{n}\right)$ 's form a base of neighbourhoods of the group unit. The completion $\Gamma_{c}$ of $\Gamma$ with respect to this topology is the projective limit

$$
\operatorname{proj} \lim \Gamma / \Gamma\left(p^{n}\right) .
$$

As is well-known, $\Gamma_{c}$ may be identified in the natural way with the closure of $\Gamma$ in $\mathbb{G}\left(\mathbb{Z}_{p}\right)$. So, by Lemma $2, \Gamma_{c}$ has finite index in $\mathbb{G}\left(\mathbb{Z}_{p}\right)$.

It is clear that the representations of $\Gamma$ that factor through some $\Gamma / \Gamma\left(p^{n}\right)$ are the ones that are continuous with respect to the topology $\tau$. Hence, these representations are exactly the restrictions to $\Gamma$ of the continuous representations of $\Gamma_{c}$. By the above, this implies that $1_{\Gamma_{c}}$ is isolated in the set $\widehat{\Gamma_{c}}$, when $\Gamma_{c}$ is viewed as a discrete group.

Let $\left(\pi_{i}\right)_{i}$ be a sequence of continuous irreducible representations of $\mathbb{G}\left(\mathbb{Z}_{p}\right)$ converging to $1_{\mathbb{G}\left(\mathbb{Z}_{p}\right)}$, where $\mathbb{G}\left(\mathbb{Z}_{p}\right)$ is endowed with the discrete topology. Since $\Gamma_{c}$ has finite index in $\mathbb{G}\left(\mathbb{Z}_{p}\right), \pi_{i}$ factors through a (fixed) finite quotient of $\mathbb{G}\left(\mathbb{Z}_{p}\right)$ and, hence, is equal to $1_{\mathbb{G}\left(\mathbb{Z}_{p}\right)}$ for $i$ large enough. This means that $1_{\mathbb{G}\left(\mathbb{Z}_{p}\right)}$ is not weakly contained in $\rho_{0}$.

We now turn to the proof of Theorem B. The following crucial fact is certainly known to the experts (compare [Ma2], Chapter III, (1.12), Remark 1). As we could not find a reference (see however [Moo], Proposition 3.6, and [BeV], Proposition 4.1 , for the case of a simple group), we shall give a proof. ${ }^{2}$

Lemma 3. Let $G$ be a semisimple real Lie group with finite centre and without compact factors, and let $\Gamma$ be a lattice in $G$. Let $\rho_{0}$ denote the restriction to $L_{0}^{2}(G / \Gamma)$ of the natural representation of $G$ on $L^{2}(G / \Gamma)$. Then $1_{G}$ is not weakly contained in $\rho_{0}$.

Proof. Let

$$
L^{2}(G / \Gamma)=L^{2}(G / \Gamma)_{\text {cont }} \oplus L^{2}(G / \Gamma)_{d i s c}
$$

be the decomposition of $L^{2}(G / \Gamma)$ into a direct sum of a discrete part $L^{2}(G / \Gamma)_{\text {disc }}$, the direct sum of all irreducible $G$-invariant subspaces in $L^{2}(G / \Gamma)$, and a continuous part $L^{2}(G / \Gamma)_{\text {cont }}$. Let $\rho_{\text {disc }}$ and $\rho_{\text {cont }}$ be the representations of $G$ defined by these subspaces.

As is well known, the theory of Eisenstein series provides a further decomposition of $\rho_{\text {cont }}$ into a direct sum of representations $\pi_{\mathcal{C}}$ associated with the (finitely many) equivalence classes $\mathcal{C}$ of proper cuspidal subgroups of $G$. Each representation $\pi_{\mathcal{C}}$ may be written as a induced representation $\pi_{\mathcal{C}}=\operatorname{Ind}_{P}^{G} \sigma$, for some representation $\sigma$ of a cuspidal subgroup $P \in \mathcal{C}$ (see [OsW], Chapter 7).

We claim that $\pi_{\mathcal{C}}$ does not weakly contain $1_{G}$. Indeed, otherwise, by Herz' majoration principle (see [EyL], p. 186 and $[\mathrm{HaS}]$, Lemme 4), $1_{G}$ would be weakly

\footnotetext{
${ }^{2}$ M. Burger (private communication) showed us how some of the arguments in the proof may be simplified in an elegant way by reducing to irreducible lattices.
} 
contained in the quasi-regular representation $\operatorname{Ind}_{P}^{G} 1_{P}$ of $G$ on $L^{2}(G / P)$. This means that $G / P$ would be amenable in the sense of Eymard; that is, there would exist a $G$-invariant mean on $L^{\infty}(G / P)$ (see [Eym]). Therefore, the compact homogeneous space $G / P$ would carry a $G$-invariant probability measure. A result of Zimmer generalizing work by Furstenberg and Moore shows that this is impossible (see [Zim], 3.2.20 Theorem). Hence the continuous part $\rho_{\text {cont }}$ does not weakly contain $1_{G}$.

As to the discrete part, by a result of Borel and Garland ([BoG], Theorem, 4.6 (i)), $\rho_{\text {disc }}$ is a so-called liminary (or CCR) representation, that is, its canonical extension to $L^{1}(G)$ acts by compact operators. The spectrum of such a representation is known to be discrete (see, e.g., [Fe1], Theorem 1.8). In particular, $1_{G}$ is isolated in $\rho_{d i s c}$, and this completes the proof.

We shall need the following fact about unitary representations of semisimple Lie groups.

Lemma 4. Let $G$ be a semisimple real Lie group with finite centre and without compact factors. Let $\pi$ be a unitary representation of $G$ that does not weakly contain the trivial representation $1_{G}$. Then, for any unitary representation $\sigma$ of $G$, the (inner) tensor product $\pi \otimes \sigma$ does not weakly contain $1_{G}$.

Proof. We first treat the case where $G$ is simple.

Suppose $G$ has property (T). If $1_{G}$ is weakly contained in $\pi \otimes \sigma$, then $1_{G}$ is contained in $\pi \otimes \sigma$. This can only happen if $\pi$ has a finite dimensional subrepresentation. This subrepresentation has to be trivial, as $G$ has no compact factor. This is a contradition, since $\pi$ does not weakly contain $1_{G}$.

Suppose $G$ is locally isomorphic to $S O(n, 1)$ or $S U(n, 1)$. It follows from the classification of the unitary dual of these groups and the asymptotic behaviour of matrix coefficient of their unitary irreducible representations that, for some integer $N$, the $N$-fold tensor product

$$
\pi^{\otimes N}=\pi \otimes \cdots \otimes \pi
$$

is contained in an infinite multiple $\infty \lambda_{G}$ of the regular representation $\lambda_{G}$ of $G$ (see [Moo], Proposition 3.6). So, $(\pi \otimes \sigma)^{\otimes N}$ is also contained in

$$
\infty \lambda_{G} \otimes \sigma^{\otimes N} \simeq \infty \lambda_{G}
$$

Hence, $1_{G}$ cannot be weakly contained in $\pi \otimes \sigma$, as $G$ is not amenable.

Now let $G$ be an arbitrary semisimple Lie group with finite centre and without compact factors. Upon replacing, if necessary, $G$ by a finite covering, we may (and will) assume that $G$ is the direct product of finitely many simple groups $G_{1}, \ldots, G_{n}$. Suppose that $1_{G}$ is weakly contained in $\pi \otimes \sigma$. Then there exist sequences of irreducible representations

$$
\pi_{1}^{(i)} \in \widehat{G_{1}}, \ldots, \pi_{n}^{(i)} \in \widehat{G_{n}}, \quad i=1,2, \ldots
$$

and

$$
\sigma_{1}^{(i)} \in \widehat{G_{1}}, \ldots, \sigma_{n}^{(i)} \in \widehat{G_{n}}, \quad i=1,2, \ldots,
$$

such that, denoting by $\times$ the outer tensor product of representations, $\pi_{1}^{(i)} \times \cdots \times \pi_{n}^{(i)}$ is weakly contained in $\pi, \sigma_{1}^{(i)} \times \cdots \times \sigma_{n}^{(i)}$ is weakly contained in $\sigma$ and

$$
\lim _{i}\left(\pi_{1}^{(i)} \times \cdots \times \pi_{n}^{(i)}\right) \otimes\left(\sigma_{1}^{(i)} \times \cdots \times \sigma_{n}^{(i)}\right)=1_{G}
$$


Hence

$$
\lim _{i}\left(\pi_{1}^{(i)} \otimes \sigma_{1}^{(i)}\right) \times \cdots \times\left(\pi_{n}^{(i)} \otimes \sigma_{n}^{(i)}\right)=1_{G}
$$

This means that

$$
\lim _{i} \pi_{1}^{(i)} \otimes \sigma_{1}^{(i)}=1_{G_{1}}, \ldots, \pi_{n}^{(i)} \otimes \sigma_{n}^{(i)}=1_{G_{n}} .
$$

For a fixed $i$, set

$$
\pi_{1}(i)=\bigoplus_{j \geq i} \pi_{1}^{j}, \quad \sigma_{1}(i)=\bigoplus_{j \geq i} \sigma_{1}^{j}, \ldots, \pi_{n}(i)=\bigoplus_{j \geq i} \pi_{n}^{j}, \quad \sigma_{n}(i)=\bigoplus_{j \geq i} \sigma_{n}^{j} .
$$

Then, by $(*), 1_{G_{k}}$ is weakly contained in $\pi_{k}(i) \otimes \sigma_{k}(i)$ for all $k=1, \ldots, n$ and all $i$. Since the $G_{k}$ are simple, the above implies that $1_{G_{k}}$ is weakly contained in $\pi_{k}(i)$ for all $i$. Hence,

$$
\lim _{i} \pi_{1}^{(i)}=1_{G_{1}}, \ldots, \lim _{i} \pi_{n}^{(i)}=1_{G_{n}} .
$$

This shows that $1_{G}$ is weakly contained in $\pi$. This contradiction completes the proof.

Proof of Theorem B. Let $\Gamma$ be a lattice in $G$, and let $\rho_{0}$ denote the restriction to $L_{0}^{2}(G / \Gamma)$ of the natural representation $\rho$ of $G$ on $L^{2}(G / \Gamma)$. By Lemma 1 , it suffices to show that $\left.\rho_{0}\right|_{\Gamma}$ does not weakly contain $1_{\Gamma}$.

Suppose, for a contradiction, that $1_{\Gamma}$ is weakly contained in $\left.\rho_{0}\right|_{\Gamma}$. Then, by continuity of inducing ([Fe2], Theorem 4.1), the quasi-regular representation $\rho=$ $\operatorname{Ind}_{\Gamma}^{G} 1_{\Gamma}$ is weakly contained in

$$
\left.\operatorname{Ind}_{\Gamma}^{G} \rho_{0}\right|_{\Gamma}=\operatorname{Ind}_{\Gamma}^{G} 1_{\Gamma} \otimes \rho_{0}=\rho \otimes \rho_{0} .
$$

As $\Gamma$ is a lattice, $L^{2}(G / \Gamma)$ contains the constants, and hence, $1_{G}$ is a subrepresentation of $\rho$. Thus, $1_{G}$ is weakly contained in $\rho \otimes \rho_{0}$. Since, by Lemma $3,1_{G}$ is not weakly contained in $\rho_{0}$, this is a contradiction to Lemma 4 . This completes the proof.

\section{ACKNOWLEDGMENT}

It is a great pleasure to thank Alain Valette for useful suggestions. He pointed out to me the problem discussed in Theorem A. Thanks are also due to Marc Burger, Pierre de la Harpe and Harald Rindler for several interesting comments.

\section{NOTE ADDED IN PROOF}

While this paper was in press, we received the interesting preprint "Expanding graphs and invariant means" by Yehuda Shalom (Hebrew University, Jerusalem) where related questions are treated. Moreover, Yehuda Shalom (private communication) showed us that the non-compactness assumption in the above Theorem A is not necessary. 


\section{REFERENCES}

$[\mathrm{BeV}]$ M. Bekka and A. Valette, Lattices in semi-simple Lie groups, and multipliers of group $C^{*}$-algebras, in Proceedings of ALGOP, Orléans, July 1992, Astérique Soc. Math. France 232 (1995), 67-79. MR 97a:22015

[BHC] A. Borel and Harish-Chandra, Arithmetic subgroups of algebraic groups, Ann. Math. 75 (1962), 485-535. MR 26:5081

[BoG] A. Borel and H. Garland, Laplacian and the discrete spectrum of an arithmetic group, Amer. J. Math. 105 (1983), 309-335. MR 84k:58220

[Bro] R. Brooks, The spectral geometry of a tower of coverings, J. Diff. Geom. 23 (1986), 97-107. MR 87j:58095

[Bur] M. Burger, Spectre du Laplacien, graphes et topologie de Fell, Comm. Math. Helv. 63 (1988), 226-252. MR 89h:58193

[BuS] M. Burger and P. Sarnak, Ramanujan duals II, Invent. Math. 106 (1993), 1-11. MR 92m:22005

[Dri] V. G. Drinfeld, Finitely additive measures on $S^{2}$ and $S^{3}$, invariant with respect to rotations, Funct. Anal. and its Appl., vol. 18, 1984, pp. 245-246. MR 86a:28012

[Dix] J. Dixmier, $C^{*}$-Algebras, North Holland, 1977. MR 56:16388

[EGM] J. Elstrodt, F. Grunewald and J. Mennicke, Kloosterman sums for Clifford algebras and a lower bound for the positive eigenvalues of the laplacian for congruence subgroups acting on hyperbolic spaces, Invent. Math. 101 (1990), 641-685. MR 91j:11038

[EyL] P. Eymard and N. Lohoué, Sur la racine carrée du noyau de Poisson dans les espaces symétriques et une conjecture de E. M. Stein, Ann. Scient. Ec. Norm. Sup. (4) 8 (1975), 179-188. MR 52:3890

[Eym] P. Eymard, Moyennes invariantes et représentations unitaires, Springer Lecture Notes in Math. 300 (1972). MR 56:6279

[Fe1] J.M.G. Fell, The dual spaces of $C^{*}$-algebras, Trans. Amer. Math. Soc. 94 (1960), 365-403. MR 26: 4201

[Fe2] J.M.G. Fell, Weak containment and induced representations of groups, Canad. J. Math. 14 (1962), 237-268. MR 27:242

[HaS] P. de la Harpe and G. Skandalis, Les réseaux dans les groupes semi-simples ne sont pas intérieurement moyennables, Ens. Math. 40 (1994), 291-311. MR 95k:22012

[Li] J.-S. Li, Kloosterman-Selberg zeta functions on complex hyperbolic spaces, Amer. J. Math. 113 (1991), 653-731. MR 92k:11055

[LoR] V. Losert and H. Rindler, Almost invariant sets, Bull. London Math. Soc. 13 (1981), 145-148. MR 82i:43001

[LPS] J.S. Li, I.I. Piatetski-Shapiro and P. Sarnak, Poincaré series for $S O(n, 1)$, Proc. Indian Acad. Sci., Math. Sci. 97 (1987), 231-237. MR 90j:11040

[Lub] A. Lubotzky, Discrete groups, expanding graphs and invariant measures, Birkhäuser Verlag, 1994. MR 96g:22018

[Ma1] G. A. Margulis, Some remarks on invariant means, Monatsh. Math. 90 (1980), 233-235. MR 82b:28034

[Ma2] G. A. Margulis, Discrete subgroups of semisimple Lie groups, Springer-Verlag, 1991. MR 92h:22021

[Moo] C.C. Moore, Exponential decay of correlations coefficients for geodesic flows, in Group Representations, Ergodic Theory, Operator Algebras, and Mathematical Physics, Proc. Conf. in honor of G.W. Mackey, MSRI Publications, Springer-Verlag (1987), 163-181. MR 89d:58102

[OsW] M. S. Osborne and G. Warner, The theory of Eisenstein systems, Academic Press, 1981. MR 83j:11034

[Ros] J. Rosenblatt, Uniqueness of invariant means for measure preserving transformations, Trans. Amer. Math. Soc. 265 (1981), 623-636. MR 83a:28026

[Sar] P. Sarnak, Some applications of modular forms, Cambridge University Press, 1990. MR 92k: 11045

[Sch] K. Schmidt, Amenability, Kazhdan's property T, strong ergodicity and invariant means for ergodic group actions, Erg. Th. Dyn. Syst. 1 (1981), 223-226. MR 83m:43001

[Sul] D. Sullivan, For $n>3$ there is only one finitely additive rotationally invariant measure on the n-sphere on all Lebesgue measurable sets, Bull. Amer. Math. Soc. 4 (1981), 121-123. MR 82b: 28035 
[Wag] S. Wagon, The Banach-Tarski Paradox, Cambridge University Press, 1985. MR 87e:04007

[Zim] R. J. Zimmer, Ergodic Theory and Semisimple Groups, Birkhäuser, 1984. MR 86j:22014

Département de Mathématiques, Université de Metz, F-57045 Metz, France

E-mail address: bekka@poncelet.univ-metz.fr 